\title{
Erratum to: On the uncertainty of interdisciplinarity measurements due to incomplete bibliographic data
}

\author{
María del Carmen Calatrava Moreno ${ }^{1}$ Thomas Auzinger ${ }^{2,3}$. \\ Hannes Werthner ${ }^{1}$
}

\section{Erratum to: Scientometrics \\ DOI 10.1007/s11192-016-1842-4}

In the original publication of the article, errors occurred in first author name, second affiliation of second author, equations and subscript terms. The correct version of those is given in this erratum.

The indices of the sums in Eq. 1 have to be written as

$$
\sum_{\substack{i=1 \\ j=1}}^{N_{\mathcal{I}}}
$$

The terms $\mathcal{T}$ prune, $\mathcal{I}$ prune, $\mathcal{G}$ span, $\mathcal{T}$ valid, $\boldsymbol{c}$ min, $\boldsymbol{c}$ max and $\mathcal{G}$ known should read as $\mathcal{T}_{\text {prune }}, \mathcal{I}_{\text {prune }}, \mathcal{G}_{\text {span }}, \mathcal{T}_{\text {valid }}, \boldsymbol{c}_{\min }, \boldsymbol{c}_{\text {max }}$ and $\mathcal{G}_{\text {known }}$.

In section "Constraint transformation lemma", the last column of the last term of the equation that starts with $\frac{\partial q_{m}}{\partial \lambda_{k}}$ is missing and the correct equation should read as

The online version of the original article can be found under doi:10.1007/s11192-016-1842-4.

\section{María del Carmen Calatrava Moreno}

calatrava@ec.tuwien.ac.at

1 E-Commerce Group, Institute of Software Technology and Interactive Systems, Vienna University of Technology, Vienna, Austria

2 Computer Graphics Group, Institute of Computer Graphics and Algorithms, Vienna University of Technology, Vienna, Austria

3 IST Austria, Klosterneuburg, Austria 


$$
\begin{aligned}
\frac{\partial q_{m}}{\partial \lambda_{k}} & =\left\{\begin{array}{cc}
u\left(\alpha\left(b_{j}-b_{k}\right)+\beta\left(a_{k}-a_{j}\right)\right)+\left(a_{k} b_{j}-a_{j} b_{k}\right)\|\mathbf{c}\|_{1}+D c_{i} & m=i \\
u\left(\alpha\left(b_{k}-b_{i}\right)+\beta\left(a_{i}-a_{k}\right)\right)+\left(a_{i} b_{k}-a_{k} b_{i}\right)\|\mathbf{c}\|_{1}+D c_{j} & m=j \\
u\left(\alpha\left(b_{i}-b_{j}\right)+\beta\left(a_{j}-a_{i}\right)\right)+\left(a_{j} b_{i}-a_{i} b_{j}\right)\|\mathbf{c}\|_{1}+D c_{k} & m=k \\
D c_{m} & m \neq i, j, k
\end{array}\right. \\
D & =\left|\begin{array}{ccc|c}
a_{i} & a_{j} & a_{k} \\
b_{i} & b_{j} & b_{k} \\
1 & 1 & 1
\end{array}\right|
\end{aligned}
$$

In section "Hypercube constraints", the terms with 'hat' notations (e.g., $\hat{\mathbf{u}})$ should read $\hat{\mathbf{u}}_{i}$ instead of $\hat{\mathbf{u}}_{i}$.

Also, the term with $\mathbf{u}_{i}$ should read as $\check{\mathbf{u}}_{i}$. 